\title{
SIMULTANEOUS RATIONAL APPROXIMATIONS TO ALGEBRAIC NUMBERS
}

\author{
BY L. G. PECK \\ Communicated by N. C. Ankeny, December 17, 1960
}

Let $K$ be an algebraic number field of degree $n+1$ over the rationals. The conjugates $K^{(0)}, K^{(1)}, \cdots, K^{(n)}$ are arranged so that $K^{(0)}, K^{(1)}, \cdots, K^{(r)}$ are real and

$$
K^{(r+s+k)}=\overline{K^{(r+k)}}, \quad(k=1,2, \cdots, s) .
$$

Here $r+2 s=n$. It will be assumed throughout that $r \geqq 0$, so that $K^{(0)}$ is real. Numbers in $K$ are denoted by Greek letters, superscripts being used for the corresponding conjugates. We shall frequently omit the superscript ${ }^{(0)}$; this identification of $K$ with $K^{(0)}$ will cause no confusion. Trace and norm of elements of $K$ are denoted by $S$ and $N$, respectively.

Let $\beta_{0}, \cdots, \beta_{n}$ be elements of $K$ which are linearly independent over the rationals. It is well known that infinitely many sets of rational integers $\left(q_{0}, q_{1}, \cdots, q_{n}\right)$ can be found satisfying,

$$
q_{0}>0, \quad \text { g.c.d. }\left(q_{0}, q_{1}, \cdots, q_{n}\right)=1,
$$

and (omitting the superscript ${ }^{(0)}$ )

$$
\left|\frac{\beta_{j}}{\beta_{0}}-\frac{q_{j}}{q_{0}}\right|<C q_{0}^{-1-1 / n}, \quad(j=1, \cdots, n),
$$

with the constant $C=1$. It will be shown here how to determine all solutions of (1), (2). From this will be deduced not only the known fact that if $C$ is too small (2) has no solutions, but also the hitherto unknown result that the sharper inequalities

$$
\begin{aligned}
\left|q_{0} \beta_{j}-q_{j} \beta_{0}\right| & <C q_{0}^{-1 / n}\left(\log q_{0}\right)^{-1 /(n-1)}, \quad(j=1, \cdots, n-1), \\
\left|q_{0} \beta_{n}-q_{n} \beta_{0}\right|<C q_{0}^{-1 / n}, &
\end{aligned}
$$

have infinitely many solutions.

This result sharpens some of the conclusions of Cassels and Swinnerton-Dyer (I), but does not furnish any further evidence for or against the conjecture of Littlewood which is considered in their paper.

A number of interesting problems can be raised in connection with (3). In one direction it can be asked whether $n-1$ of the inequalities (2) can be improved with factors which are not all the same; e.g., 
one might conjecture that we can find infinitely many solutions of the inequalities

$$
\begin{aligned}
\left|q_{0} \beta_{j}-q_{j} \beta_{0}\right| & <C q_{0}^{-1 / n} / f_{j}\left(q_{0}\right), \\
\left|q_{0} \beta_{n}-q_{n} \beta_{0}\right| & <C q_{0}^{-1 / n},
\end{aligned} \quad(j=1, \cdots, n-1),
$$

with $f_{1}\left(q_{0}\right) \cdots f_{n-1}\left(q_{0}\right)=\log q_{0}$ and $f_{j}\left(q_{0}\right) \geqq 1(j=1, \cdots, n-1)$.

A much more difficult set of problems is in the direction of the Thue-Siegel-Roth theorem, in which one tries to specify the functions $f_{j}$ in such a way that the corresponding inequalities have at most a finite number of solutions. In view of Roth's theorem one might conjecture that $f_{j}=q_{0}^{-\mathrm{e}}$ would have the indicated effect, but this is by no means obvious.

The numbers $\beta_{0}, \cdots, \beta_{n}$ form the basis of a module $M$. Denote by $R$ the set of all integers $\rho$ in $K$ such that $\rho \beta$ is in $M$ whenever $\beta$ is in $M$. Clearly $R$ is a ring. By the Dirichlet theory of units, we may find a basis $\epsilon_{1}, \cdots, \epsilon_{r+s}$ of the units in $R$. Since the only roots of unity in $K$ are \pm 1 (because $K^{(0)}$ is real) every unit $\epsilon$ in $R$ is uniquely expressible in the form

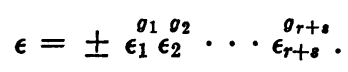

Let $C_{1}=\max _{i, j=1, \ldots, r+s}|\log | \epsilon_{j}^{(i)}||$. Then, for any real number $T$ we can find integers $g_{1}, \cdots, g_{r+s}$ such that

$$
\begin{array}{r}
-n^{-1} T-\frac{1}{2} C_{1} \leqq \sum_{j=1}^{r+s} g_{j}\left|\log \epsilon_{j}^{(i)}\right|<-n^{-1} T+\frac{1}{2} C_{1}, \\
(i=1, \cdots, r+s),
\end{array}
$$

and, since $N\left(\epsilon_{j}\right)=1$,

$$
T-\frac{n}{2} C_{1}<\sum_{j=1}^{r+s} g_{j}\left|\log \epsilon_{j}\right| \leqq T+\frac{n}{2} C_{1} .
$$

Using (4) with the sign chosen so that $\epsilon>0$, we obtain

$$
\left|\epsilon^{(i)}\right|<C_{2} \epsilon^{-1 / n}
$$

with a constant $C_{2}=e^{C_{1}}$ which depends only on the ring $R$. A unit $\epsilon>1$ which satisfies (5) will be called dominant. We have proved that for every real $T>1$ there is a dominant unit $\epsilon$ satisfying $T \leqq \epsilon<C_{2}^{n} T$.

The elements $\delta$ of $K$, such that $S(\delta \beta)=$ a rational integer for every $\beta$ in $M$, form another module $D$. A basis $\delta_{0}, \delta_{1}, \cdots, \delta_{n}$ of $D$ is obtained by solving the equations 


$$
S\left(\beta_{i} \delta_{j}\right)=\left\{\begin{array}{ll}
1, & (i=j) \\
0, & (i \neq j)
\end{array} \quad(i, j=0, \cdots, n) .\right.
$$

Because of the discreteness of $D$, there is, among the nonzero elements of $D$, one whose norm has minimal absolute value; this minimal norm will be denoted by $v$. Note also that if $\rho$ is in $R$ and $\delta$ in $D$ then $\rho \delta$ is in $D$.

Choose $\delta=a_{0} \delta_{0}+a_{i} \delta_{i}+\cdots+a_{n} \delta_{n}$ in $D$ so that $\delta \beta_{0}>0$ and g.c.d. $\left(a_{0}, a_{i}, \cdots, a_{n}\right)=1$. If $\epsilon$ is a unit in $R$ and $\epsilon \delta=q_{0} \delta_{0}+q_{1} \delta_{1}+\cdots$ $+q_{n} \delta_{n}$ we must have g.c.d. $\left(q_{0}, \cdots, q_{n}\right)=1$. For if g.c.d. $\left(q_{0}, \cdots, q_{n}\right)$ $=q$, it is clear that $q^{-1} \epsilon \delta$ is in $D$, whence $\epsilon^{-1} q^{-1} \epsilon \delta=q^{-1} \delta$ is in $D$ and $q$ divides g.c.d. $\left(a_{0}, \cdots, a_{n}\right)=1$.

As defined above, we have

$$
q_{k}=S\left(\epsilon \delta \beta_{k}\right), \quad(k=0, \cdots, n) .
$$

Thus, if we assume that $\epsilon$ is dominant, we have

$$
\begin{aligned}
\left|q_{k} \beta_{0}-q_{0} \beta_{k}\right| & =\left|\sum_{j=1}^{n}\left(\beta_{k}^{(j)} \beta_{0}-\beta_{k} \beta_{0}^{(j)}\right) \delta^{(j)} \epsilon^{(j)}\right| \\
& <C_{3} \epsilon^{-1 / n}, \quad(k=1, \cdots, n),
\end{aligned}
$$

while

$$
\left|q_{0}-\epsilon \delta \beta_{0}\right|=\left|\sum_{j=1}^{n} \epsilon^{(j)} \delta^{(j)} \beta_{0}^{(j)}\right|<C_{4} \epsilon^{-1 / n} .
$$

The last two inequalities imply (2). The constants $C_{3}, C_{4}, C$ depend on $a_{0}, \cdots, a_{n}$, but we may remove this dependence if the choice of $\delta$ is made from a fixed bounded region.

Suppose conversely that (1) and (2) hold (with some $C>0$ ). Define $\zeta=q_{0} \delta_{0}+\cdots+q_{n} \delta_{n}$, so that $\zeta$ is in $D$. We have

$$
\zeta^{(i)}=\frac{1}{\beta_{0}} \sum_{j=1}^{n}\left(q_{j} \beta_{0}-q_{0} \beta_{j}\right) \delta_{j}^{(i)}+\frac{q_{0}}{\beta_{0}} \sum_{j=0}^{n} \beta_{j} \delta_{j}^{(i)}, \quad(i=0, \cdots, n) .
$$

It follows easily from (6) that the last sum is 1 or 0 according as $i=0$ or $i \neq 0$. Thus

$$
\left|\zeta-\frac{q_{0}}{\beta_{0}}\right|-C q_{0}^{-1 / n} \sum_{j=1}^{n}\left|\delta_{j}\right|
$$

while

$$
\left|\zeta^{(i)}\right|<C q_{0}^{-1 / n} \sum_{j=1}^{n}\left|\delta_{j}^{(i)}\right|, \quad(i=1, \cdots, n)
$$


Choose a dominant unit $\epsilon$ such that $\left|q_{0} / \beta_{0}\right| \leqq \epsilon<C_{2}^{n}\left|q_{0} / \beta_{0}\right|$ and set $\delta= \pm \epsilon^{-1} \xi$ with the sign chosen so that $\delta>0$. Then

$$
0<\delta<1+C\left|\beta_{0}\right|^{-1} q_{0}^{-1-1 / n} \sum_{j=1}^{n}\left|\delta_{j}\right|,
$$

while

Thus

$$
0<\left|\delta^{(i)}\right|<C\left|\epsilon^{(i)}\right|^{-1} q_{0}^{-1 / n} \sum_{j=1}^{n}\left|\delta_{j}^{(i)}\right|
$$

$$
\begin{aligned}
0<|N(\delta)| & <C^{n} \epsilon q_{0}^{-1} \prod_{i=1}^{n} \sum_{j=1}^{n}\left|\delta_{j}^{(i)}\right|\left(1+O\left(q_{0}^{-1-1 / n}\right)\right) \\
& <\left(C C_{2}\right)^{n} C_{4},
\end{aligned}
$$

where $C_{4}$ depends only on $\beta_{0}, \cdots, \beta_{n}$. It follows that $\delta$ is an element of $D$ which lies in a bounded region (which will be vacuous if $\left.C \leqq v^{1 / n} / C_{2} C_{4}^{1 / n}\right)$ and that the $q_{k}$ are given by (7).

The proof of (3) is based on a special choice of $\delta$ in (7) together with a sharper form of (5) for a certain infinite set of dominant units.

To obtain the latter, let

$$
\epsilon_{k}^{(j)}=\left\{\begin{array}{lr}
\epsilon_{k}^{-1 / n} e^{\phi j k} e_{j k}, & (j=1, \cdots, r), \\
\epsilon_{k}^{-1 / n} e^{\phi j k+2 i \pi \psi j k}, & (j=r+1, \cdots, r+s),
\end{array}\right.
$$

where $\phi_{j k}$ and $\psi_{j k}$ are real and $e_{j k}= \pm 1$.

If the dominant unit $\epsilon$ is given by (4) we have

$$
\left|\sum_{k=1}^{r+s} \phi_{j k} g_{k}\right|=|\log | \epsilon^{1 / n} \epsilon^{(j)}||<C_{1}, \quad(j=1, \cdots, r+s) .
$$

Also, we can find rational integers $h_{j}$ such that

$$
\left|(2 \pi)^{-1} \arg \epsilon^{(j)}\right|=\left|\sum_{k=r+1}^{r+s} \psi_{j k} g_{k}+h_{j}\right| \leqq 1 / 2 .
$$

Now there are at least $M+1$ distinct dominant units $\epsilon$ in the interval $1 \leqq \epsilon<e^{(M+1) n C_{1}}$. By the well known schubfachprinzip of Dirichlet we may therefore find two-call them $\eta$ and $\theta$ - such that $1 \leqq \theta<\eta$ $<e^{(M+1) n C_{1}}$,

$|\log | \eta^{1 / n} \eta^{(j)}|-\log | \theta^{1 / n \theta^{(j)}}||<2 C_{1} / M^{1 /(n-1)},(j=2, \cdots, r+s)$

and

$$
\pi^{-1}\left|\arg \eta^{(j)}-\arg \theta^{(j)}\right| \leqq M^{-1 /(n-1)}, \quad(j=r+1, \cdots, r+s) .
$$


Thus the unit $\epsilon=\eta / \theta$ satisfies $1<\epsilon<C_{2}^{n} T$ (where $T=e^{M n C_{1}}$ ) and

$$
\begin{array}{rlrl}
|\log | \epsilon^{1 / n} \epsilon^{(j)} \mid & <2\left(C_{1}^{n} n / \log T\right)^{1 /(n-1)}, & & (j=2, \cdots, r+s), \\
\left|\arg \epsilon^{(j)}\right| & \leqq 2 \pi\left(C_{1} n / \log T\right)^{1 /(n-1)}, & (j=r+1, \cdots, r+s) .
\end{array}
$$

Moreover, since

$$
\sum_{j=1}^{r} \log \left|\epsilon^{1 / n} \epsilon^{(j)}\right|+2 \sum_{j=r+1}^{r+8} \log \left|\epsilon^{1 / n} \epsilon^{(j)}\right|=0,
$$

we have also

$$
|\log | \epsilon_{\epsilon}^{1 / n(1)}||<2(n-1)\left(C_{1}^{n} n / \log T\right)^{1 /(n-1)} .
$$

It follows that

$$
\begin{array}{r}
\epsilon^{(j)}=\left|\epsilon^{(j)}\right| \exp \left(i \arg \epsilon^{(j)}\right)=\epsilon^{-1 / n}\left(1+O(\log T)^{-1 /(n-1)}\right), \\
(j=1, \cdots, r+s),
\end{array}
$$

which is the required refinement of (5).

If we choose $\delta=\delta_{n}$ in (7) and make use of (6) and (9) we can improve (8) as follows:

$$
\begin{aligned}
\left|q_{k} \beta_{0}-q_{0} \beta_{k}\right| & =\epsilon^{-1 / n}\left(\left|\sum_{j=1}^{n}\left(\beta_{k}^{(j)} \beta_{0}-\beta_{k} \beta_{0}^{(j)}\right) \delta_{n}^{(j)}\right|+O(\log T)^{-1 /(n-1)}\right) \\
& =\left\{\begin{array}{cr}
O\left(\epsilon^{-1 / n}(\log T)^{-1(n-1)}\right) & (k=1, \cdots, n-1) \\
O\left(\epsilon^{-1 / n}\right) & (k=n) .
\end{array}\right.
\end{aligned}
$$

This, together with $1<\epsilon<C_{2}^{n} T$, implies (3).

\section{REFERENCE}

(I) J. W. S. Cassels and H. P. F. Swinnerton-Dyer, On the product of three homogeneous linear forms and indefinite ternary quadratic forms, Philos. Trans. Roy. Soc. London. Ser. A, vol. 248 (1955) pp. 73-96.

Boston, Massachusetrs 